nomials in $y$, viz. $P_{1}^{(k)}, P_{2}^{(k)}, \ldots, P_{N}^{(k)}$ such that $f_{1}^{(k)}: f_{2}^{(k)}: \ldots$ $: f_{N}^{(k)}=P_{1}^{(k)}: P_{2}^{(k)}: \cdots: P_{N}^{(k)}$, there will exist such polynomials in which the lowest powers of $y$ are respectively equal to the lowest powers of $y$ in the series $f_{1}^{(k)}, f_{2}^{(k)}, \ldots, f_{N}^{(k)}$. Hence there will exist a series $\Phi^{(0)}(y)$ with non-vanishing constant term satisfying (6). It is then easy to construct the polynomial $P^{(k)}$ satisfying the relation $\Phi^{(0)} f^{(k)}=P^{(k)}$. In view of the homogeneity of $f$ this relation may be written in the form $\Phi f=P$. From this may be obtained the equivalent form (1).

If $f\left(y ; x_{1}, \ldots, x_{n}\right)$ is not homogeneous in $x_{1}, x_{2}, \ldots, x_{n}$ the condition of the theorem is not sufficient.

The University of Saskatchewan

\title{
THE CLASS NUMBER RELATIONS IMPLICIT IN THE DISQUISITIONES ARITHMETICAE*
}

BY E. T. BELL

1. Introduction. The point of this note is its moral, which is to the effect that in arithmetic attention to trifles sometimes leads to beautiful and recondite truths. In particular, certain important expansions of Kronecker and Hermite relating to the number $F(n)$ of uneven classes of binary quadratic forms of negative determinant $-n$ are implicit in $\S 292$ of the Disquisitiones Arithmeticae of Gauss, and might have been read off from there at a glance by anyone familiar with the Fundamenta Nova of Jacobi, thirty years before Kronecker first came upon them by the devious route of complex multiplication. The relevant trifle in this instance is changing the sign of an arbitrary constant throughout an algebraic identity.

\footnotetext{
* Presented to the Society, April 5, 1924.
} 
It is well known that the tenth of Kronecker's relations* is equivalent to his expansion

(XI) $12 \sum_{0}^{\infty} E(n) q^{n}=\left[\sum_{-\infty}^{\infty} q^{n^{2}}\right]^{3}=\vartheta_{3}^{3}(q), E(n)=F(n)-F_{1}(n)$,

where $F_{1}(n)$ is the number of even classes, which is merely the algebraic expression of the theorem of Gauss (loc. cit.) on the number of representations of $n$ as a sum of three squares; and it has recently been remarked by Mordellt that Hermite's important formula

$$
\sum_{0}^{\infty} F(8 n+3) q^{8 n+3}=\left(q+q^{9}+q^{25}+\cdots\right)^{3}=\frac{1}{8} \vartheta_{2}^{3}\left(q^{4}\right),
$$

and those of Kronecker (changed from $k, K$ to $\vartheta$ notation),

$$
\begin{aligned}
& 4 \sum_{0}^{\infty} F(4 n+2) q^{4 n+2}=\vartheta_{3}\left(q^{4}\right) \vartheta_{2}^{2}\left(q^{4}\right), \\
& 4 \sum_{0}^{\infty} F(4 n+1) q^{4 n+1}=\vartheta_{2}\left(q^{4}\right) \vartheta_{3}^{2}\left(q^{4}\right),
\end{aligned}
$$

are implicit in (XI) and can be obtained from it by elementary algebra. Note in passing that (1) is simply the algebraic translation of $\S 293$ of the Disquisitiones Arithmeticae. Both Kronecker and Hermite made much use of (1), (2), (3) in their successive simplification of the classical relations and in further deductions, and these identities reappear repeatedly in works of later writers. It.is interesting therefore to see that (1), (2), (3) follow from (XI) even more simply than as shown by Mordell; in fact they result from (XI) by changing the sign of $q$. That is, (XI), (1), (2), (3) are immediate consequences of Gauss' theorem. The deduction of class number relations from these four identities by combining them with the expansions of Jacobi is not only classical but obvious, and it seems surprising that the immediate step of translating the application which Gauss made of his theorem, to representations as

* Cf. Dickson's History, vol. 3, pp. 108, 109, 113.

$\dagger$ Messenger of Mathematics, vol. 45 (1915) pp. 78, 79. 
sums of four squares, into the equivalent class number relation was not taken much earlier than it was.

2. Consequences of Gauss' Theorem. In (XI) change $q$ into $-q$ :

$\left(\mathrm{XI}_{1}\right) \quad 12 \sum_{0}^{\infty}(-1)^{n} E(n) q^{n}=\left[\sum_{-\infty}^{\infty}(-1)^{n} q^{n^{2}}\right]^{3}=\vartheta_{0}^{8}(q)$.

Let $n_{1}, n_{2}, \ldots$ denote arbitrary integers $\gtreqless 0, m_{1}, m_{2}, \ldots$ odd integers $\gtrless 0$, and write $N\left(n=x^{2}+y^{2}+z^{2}\right)$ for the number of integer solutions $x, y, z \gtreqless 0$ of $n=x^{2}+y^{2}+z^{2}$. Equate coefficients of $q^{n}$ in $\left(\mathrm{XI}_{1}\right)$ :

$12(-1)^{n} E(n)$

$$
\begin{aligned}
& \quad=N\left(n=4 n_{1}^{2}+4 n_{2}^{2}+4 n_{\mathrm{s}}^{2}\right)+N\left(n=4 n_{1}^{2}+m_{2}^{2}+m_{\mathrm{s}}^{2}\right) \\
& +N\left(n=m_{1}^{2}+4 n_{2}^{2}+m_{3}^{2}\right)+N\left(n=m_{1}^{2}+m_{2}^{2}+4 n_{3}^{2}\right) \\
& -N\left(n=m_{1}^{2}+m_{2}^{2}+m_{3}^{2}\right)-N\left(n=m_{1}^{2}+4 n_{2}^{2}+4 n_{3}^{2}\right) \\
& -N\left(n=4 n_{1}^{2}+m_{2}^{2}+4 n_{3}^{2}\right)-N\left(n=4 n_{1}^{2}+4 n_{2}^{2}+m_{8}^{2}\right), \\
& \quad=N\left(n=4 n_{1}^{2}+4 n_{2}^{2}+4 n_{3}^{2}\right)+3 N\left(n=4 n_{1}^{2}+m_{2}^{2}+m_{3}^{2}\right) \\
& \quad-N\left(n=m_{1}^{2}+m_{2}^{2}+m_{3}^{2}\right)-3 N\left(n=m_{1}^{2}+4 n_{2}^{2}+4 n_{3}^{2}\right) . \\
& \text { Since }
\end{aligned}
$$

$$
\begin{array}{r}
4 n_{1}^{2}+4 n_{2}^{2}+4 n_{3}^{2} \equiv 0 \bmod 4, \\
4 n_{1}^{2}+m_{2}^{2}+m_{3}^{2} \equiv 2 \bmod 4, \\
m_{1}^{2}+m_{2}^{2}+m_{3}^{2} \equiv 3 \bmod 8, \\
m_{1}^{2}+4 n_{2}^{2}+4 n_{3}^{2} \equiv 1 \bmod 4,
\end{array}
$$

we have $12 E(n)=N\left(4 n=4 n_{1}^{2}+4 n_{2}^{2}+4 n_{3}^{2}\right)$, which is merely (XI) again, and

$$
\begin{gathered}
N\left(8 n+3=m_{1}^{2}+m_{2}^{2}+m_{3}^{2}\right)=12 E(8 n+3), \\
N\left(4 n+2=4 n_{1}^{2}+m_{2}^{2}+m_{3}^{2}\right)=4 E(4 n+2), \\
N\left(4 n+1=m_{1}^{2}+4 n_{2}^{2}+4 n_{3}^{2}\right)=4 E(4 n+1) ;
\end{gathered}
$$

which are equivalent respectively to

$$
\begin{aligned}
\left(\sum q_{1}^{m_{1}^{2}}\right)^{3} & =12 \sum q^{8 n+8} E(8 n+3), \\
\sum q^{4 n_{1}^{2}} \times\left(\sum q_{1}^{m_{1}^{2}}\right)^{2} & =4 \sum q^{4 n+2} E(4 n+2), \\
\sum q^{m_{1}^{2}} \times\left(\sum q^{4 n_{1}^{2}}\right)^{2} & =4 \sum q^{4 n+1} E(4 n+1) .
\end{aligned}
$$

But these are identical respectively with (1), (2), (3), since $E(8 n+3)=\frac{2}{8} F(8 n+3), E(4 n+2)=F(4 n+2)$,

The University of Washington

$$
E(4 n+1)=F(4 n+1) \text {. }
$$

